\title{
Correlation of Firing Pin Impressions Based on Congruent Matching Cross-sections (CMX) Method
}

\begin{abstract}
Comparison of firing pin impressions of cartridge cases is an important part of firearms evidence identification. However, compared with breach face impressions, there is only a limited surface area over which firing pin impressions can be compared. Furthermore, the curvature of firing pin impressions makes it difficult to perform automatic correlations of the surfaces. In this study, a new method and related algorithm named congruent matching cross-sections $(\mathrm{CMX})$ are proposed. Each firing pin impression is sliced into layers and the resulting circular cross-sections are converted to two dimensional linear profiles by a polar coordinate transformation. The differential profile extraction method is used for extracting the high frequency micro-features, or the individual characteristics, for accurate correlation. Three parameters are proposed for determining whether these pairwise firing pin impressions are fired from the same firearm. The crosscorrelation function $(\mathrm{CCF})$ is used for quantifying similarity of the pairwise profiles which represent the two correlated firing pin images. If the correlated cartridge pair is fired from the same firearm, the maximum CCF value between each of the profile pairs from the reference and the correlated firing pin impressions will be high. The other two parameters relate to the horizontal (or angular) and vertical range of relative shifts that the profiles undergo to obtain the maximum CCF. These shifts are the phase angle $\theta$ which corresponds to a horizontal shift of the $2 \mathrm{D}$ profiles and the vertical shift distance of slice section, i.e. where the profiles match in the depth of the impression. These shift parameters are used to determine the congruency of the pairwise profile patterns. When these parameter values and their statistical distributions are collected for analysis, the CMX number is derived as a key parameter for a conclusive identification or exclusion. Validation tests using 40 cartridge cases of three different brands fired from 10 firearms produced by three different manufacturers yielded clear separation between known matching (KM) and known non-matching (KNM) image pairs, which strongly supports the effectiveness and feasibility of the proposed CMX method.
\end{abstract}

Keywords: forensic science; firearm identification; firing pin impression; congruent matching crosssections; CMX; cross-correlation function; CCF.

\section{Introduction}

Firearm identification by analysis of ballistics evidence under a comparison microscope has a long history in the United States. However, the scientific foundation of firearm and 
toolmark identification has been challenged by the 2009 National Academies Report [1]. This report made it a national priority in forensic science to establish a scientific foundation and a statistical procedure for quantitative error rate reports to support firearm identifications, in the same way that reporting procedures have been established for forensic identification of DNA evidence [1].

The congruent matching cells (CMC) method was recently invented at the National Institute of Standards and Technology (NIST) for accurate image-related forensic evidence identification and error rate estimation $[2,3]$. The CMC method is based on the principle of discretization-it divides the entire image into small correlation regions, and uses multiple identification parameters with corresponding thresholds for accurate forensic evidence identification and error rate estimation [3]. The pairwise cell correlations can identify the "valid correlation area" and eliminate the "invalid correlation area" for accurate and robust ballistics identification [2]. The use of multiple identification parameters for the multiple pairwise cell correlations between the evidence and the reference images makes it possible to develop an error rate procedure based on statistical analysis of the total number of correlation cells, the number of the congruent matching cells, and the statistical distributions of the identification parameters [3]. Based on the CMC method, a statistical model was developed at NIST for error rate reporting of ballistics identifications [3]. An initial test report for error rate estimation of breech face correlations using the CMC method has passed NIST review for publication [4].

Initial validation for the CMC method was conducted using 40 cartridge cases ejected from guns with 10 consecutively manufactured pistol slides. These samples generated 780 topography comparisons using the CMC method which did not produce any false identifications or false exclusions [5,6]. However, when the CMC method is used to compare firing pin impressions, challenges arise from the small correlation area and the curvature shape of the firing pin impressions. Unlike the breech face impressions with a flat 
surface and several $\mathrm{mm}^{2}$ correlation area, the firing pin impression is a concave shape with a smaller correlation area $\left(1 \mathrm{~mm}^{2}\right.$ or smaller). As a result, when the firing pin impression is divided into correlation cells, each cell may be too small to contain enough individual characteristics (topography peaks, valleys...) for accurate ballistics identification.

Based on the same congruent matching theory, the congruent matching cross-sections (CMX) method is proposed. In the following sections, we introduce the basic concept of the CMX method in Section 2; we discuss the detailed procedure, algorithm in Section 3; and demonstrate experiments and results in Section 4.

\section{Congruent matching cross-sections (CMX) method}

As mentioned above, due to the small area and concave shape of firing pin impressions, the congruent matching cross-sections (CMX) method was developed. A cross-section refers to a closed curve obtained by slicing the firing pin impression at an arbitrary height. As shown in Fig.1 (a), if slices are obtained at specific intervals in the vertical direction, a set of cross-sections from a firing pin impression can be acquired.

In Fig. 1 (b), by means of the polar coordinate transformation [7], these circular sections can be converted to a set of two dimensional linear profiles which represent the original impression uniquely. The phase of these linear profiles corresponds to an angular position of the circular profiles. Then, the cross-correlation function (CCF) was calculated for pairwise profiles from the same altitude of the two compared impressions. During CCF calculation, the pairwise linear profile should be assumed periodic so that this calculation can be done over all phases. Ideally, the profiles from the same vertical slice of two cartridge cases fired by the same firing pin should have a high correlation score when registered at the same phase angle $\theta$. The horizontal shift distance of the phase angle $\theta$ and vertical shift distance of the slice location are used to determine the congruency of the pairwise correlated profile patterns. We define the CMX number as the number of potentially congruent profile pairs. If this CMX number is equal to or more than a designed 
identification criterion, these casings are identified as a 'matching pair'.

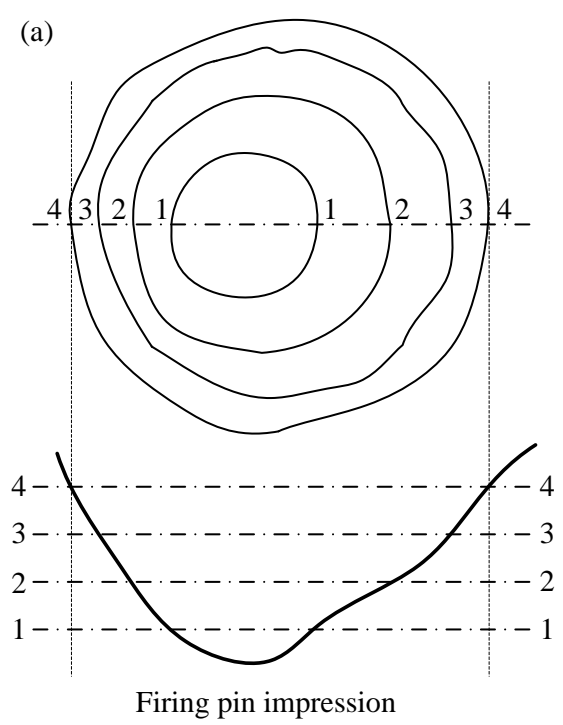

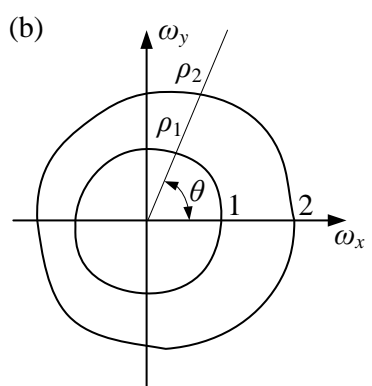

Polar coordinate transformation

Fig. 1. Sketch of acquisition of cross-sections and profiles: (a) slicing; (b) polar coordinate transformation.

\section{Methods}

\subsection{Topography measurement and data processing}

Topography measurements were performed with a Nanofocus $\mu$ Surf* disk-scanning confocal microscope to produce topography images of the impressions. White light from a xenon bulb source enters through the objective of the microscope and illuminates the surface. The light reflects back into the objective and is directed onto a pinhole. Only the light reflected back from the current focal plane can focus through the pinhole and onto the detector. The microscope scans through a range of z-slices or focal heights during the acquisition. At the end, all the slices are compiled into a three-dimensional topography map [8]. Figure 2 shows a typical topography image of the firing pin impression. A single field of view of $20 \times$ objective is square with approximate dimensions $0.8 \mathrm{~mm} \times 0.8 \mathrm{~mm}$. The raw topography images have $1.56 \mu \mathrm{m} X / \mathrm{Y}$ resolution and consist of $512 \times 512$ pixels. The $\mathrm{z}$-slice interval is 0.2 to $0.3 \mu \mathrm{m}$ and about 400 image slices are measured for a total vertical range of $120 \mu \mathrm{m}$.

\footnotetext{
* Certain commercial equipment, instruments, or materials are identified in this paper to foster understanding. Such identification does not imply recommendation or endorsement by the National Institute of Standards and Technology, nor does it imply that the materials or equipment identified are necessarily the best available for the purpose.
} 


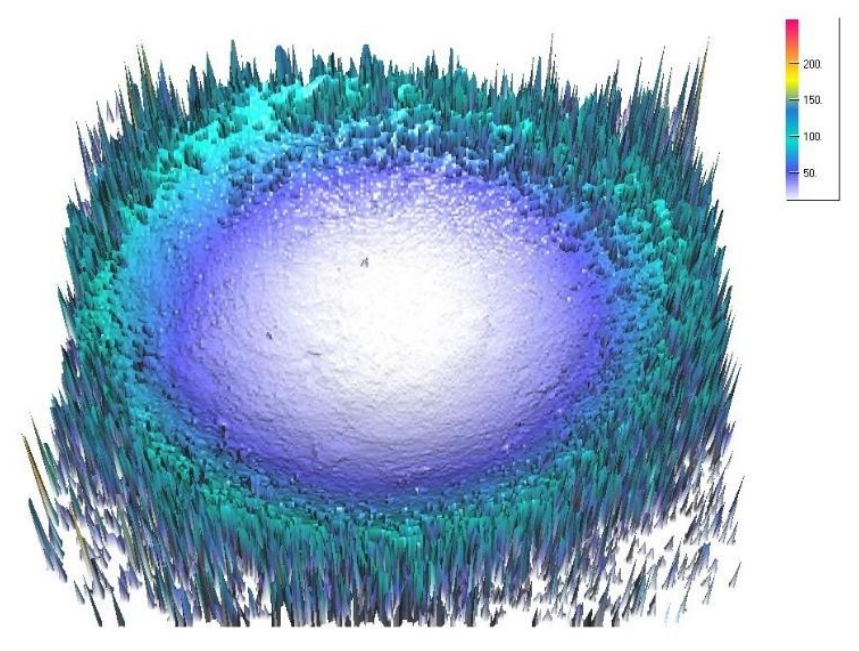

Fig. 2. Typical topography image of a firing pin impression.

The raw data acquired by the confocal microscope is bound to contain unreliable components including noise, dropouts and outliers. After acquisition, a data processing procedure was performed consisting of four steps:

(a) Identify and remove dropouts and outliers. The criterion for identifying outliers and dropouts is the same used in reference [8]. Figure 3 shows the firing pin topography data after identifying and removing datapoints of dropouts and outliers from the raw topography image of Fig. 2.

(b) Apply a low-pass spline filter with a short cutoff wavelength $\lambda_{1}$ to remove high frequency noise [9].

(c) Utilize a low-pass Gaussian regression filter [10] or the robust spline filter [9] with a relatively longer short cutoff wavelength $\lambda_{2}$ to obtain a mean surface.

(d) In the surface obtained in step (b), replace all unreliable points identified in step (a) with values of the mean surface calculated in step (c).

The purpose of the above steps is to remove the unreliable data points, and to provide an intact and robust topography image as well as its mean surface for the cross-section extraction process in the next. After testing different short- and long-cutoff wavelengths, the short cutoff wavelength for the low-pass spline filter was chosen to be $\lambda_{1}=5$ pixels (corresponding to $7.81 \mu \mathrm{m}$ ) and the long cutoff wavelength for the robust filter, used to 
calculate the mean surface, was chosen to be $\lambda_{2}=60$ pixels (corresponding to $93.75 \mu \mathrm{m}$ ).

Figure 4 shows the firing pin impression of Fig. 3, after the low-pass-filtering, processing, and remedying unreliable data points.

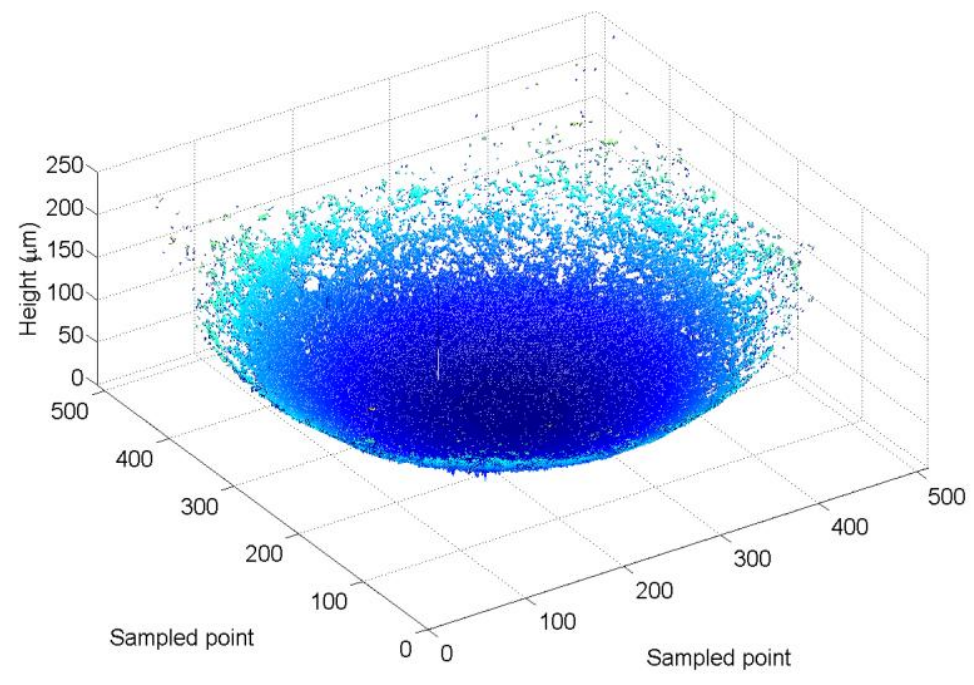

Fig. 3. Firing pin impression after dropout and outlier identification.

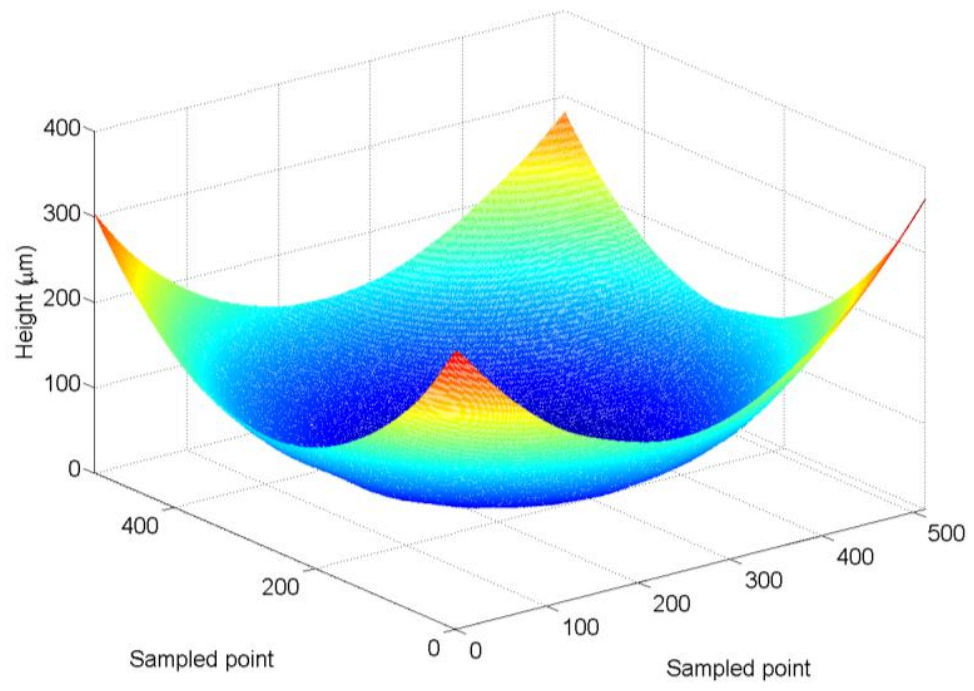

Fig. 4. Firing pin impression after the low-pass-filtering, processing, and remedying unreliable data points.

\subsection{Cross-section extraction}

A practical cross-section extraction algorithm is developed for the CMX method. The general procedure is to assign a height interval, divide the firing pin impression into slices at different altitudes, and obtain a set of cross-sections. For the initial testing, the reference plane is chosen to be at the bottom of the firing pin impression, and the height interval is 
selected to be $0.2 \mu \mathrm{m}$. Figures 5 (a) and (b) show a raw cross-section example obtained by slicing at an arbitrary altitude. Ideally, a cross-section is obtained by counting only the points occurring at the same vertical height. However, due to valleys or peaks existing on the firing pin impression, as well as the random instrument noise, it is difficult to extract a fully enclosed cross-section only by the slicing method. In fact, a raw cross-section after slicing most often presents a kind of chipped edge like a saw-tooth, especially that at the bottom of firing pin images, which may affect correlation accuracy.
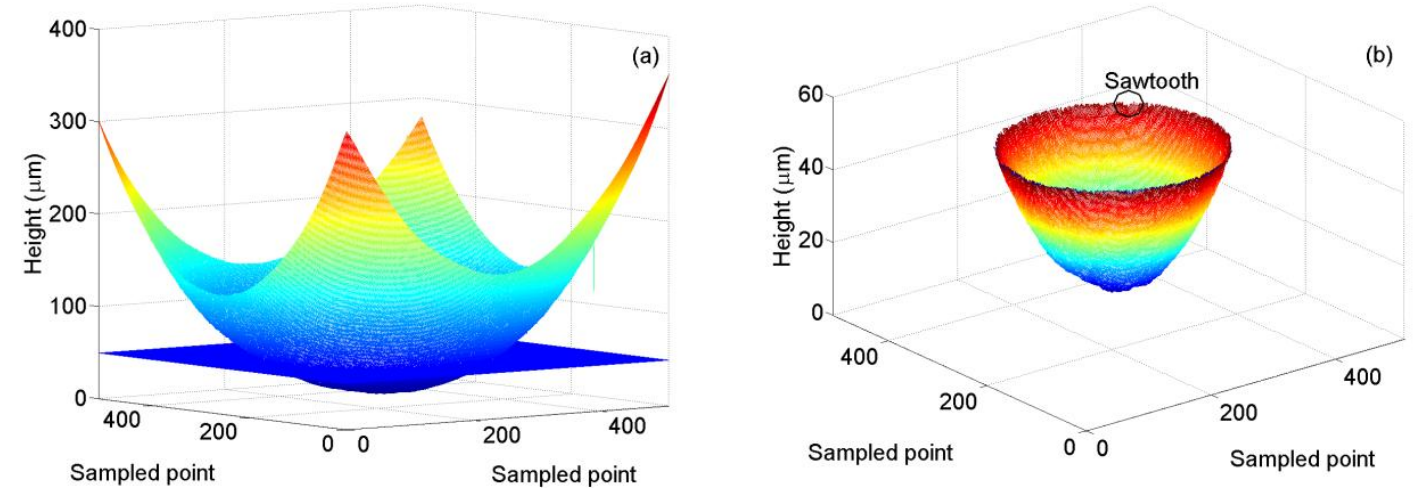

Fig. 5. Slicing a firing pin impression at an arbitrary altitude: (a) slicing process; (b) a raw cross-section example obtained by slicing.

A better way to produce the enclosed radial profile, at a particular height, is to use edge detection methods. The three dimensional cross-section of Fig. 5 (b) is converted into a binary image. This is achieved by setting the height of all pixels in the image which have vertical values less than the specified height level of the slice to a value of one. The height of all the other pixels are set to zero. In this way, the three dimensional profile of Fig. 5 (b) is projected onto a two dimensional binary image as shown in Fig. 6 (a). During this process, some small holes may occur within the object's solid zone. These holes are detected and filled in the binary image [11]. Although the outline in Fig. 6 (a) seems quite distinct, peaks and valleys on the surface are often prone to distort the desirable boundary curve. The boundary can be further cleaned and smoothed using the binary erosion and dilation procedure with a small radius parameter $[11,12]$. 


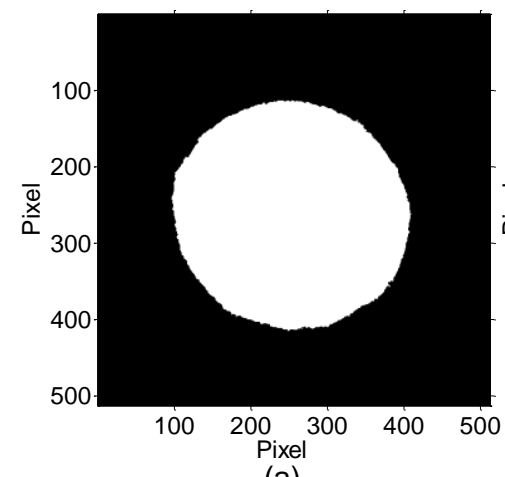

(a)

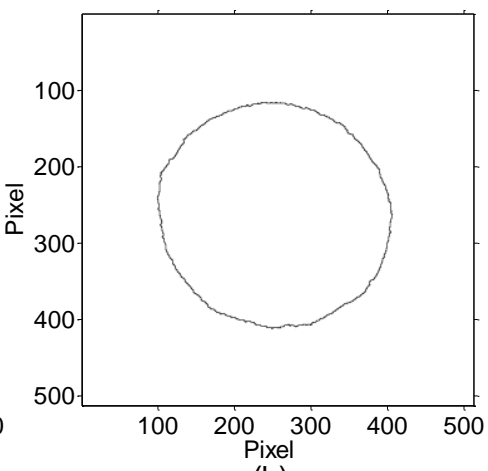

(b)

Fig. 6. A cross-section extraction: (a) binary image; (b) edge detection.

The final contour curve in the image is identified by recording only the perimeter pixels of the solid circle, as shown in Fig. 6 (b). This curve represents the extracted section which will be correlated with the extracted section from another firing pin impression.

\subsection{Differential profile extraction}

For two extracted sections, direct comparison is sometimes impossible because of different radii or outline. The sections can be transformed into profiles so as to facilitate a quantitative comparison. For each circular cross-section from the mean surface, the least squares circle method is applied to locate its center point. Averaging the horizontal coordinates of these center points tends to determine a common center. Using this point as the origin, the contour curves of the original topography after the noise filter and the mean surface at the same altitude are converted to a linear profile pair with the polar coordinate transformation shown in Fig. 1 (b). An example of this process is shown in Figs 7. (a) and (b), where profiles are from the original topography and the mean surface, respectively. In our study, each cross-section is transformed to a profile with 720 points, which indicates a 0.5 degree angle step interval during the polar coordinate transformation. It should be noted that the profiles are obtained at a fixed angle resolution so depending on the radius of the cross-section, they correspond to different spatial linear resolution. Moreover, not all crosssections contain enough data points, especially those close to the bottom of the firing pin impression. Interpolation is a necessary and convenient way to guarantee enough data 
points.

Conceptually, when two cartridge cases are fired from the same firearm, their firing pin impressions were impressed by the same firing pin, and therefore, have similarities both in their macro- and micro-geometric features. The macro-features, or the size, shape, curvature and waviness, represent the class (and sub-class) characteristics, which are common to certain brand of firearms. These features can be used to exclude a firearm during a comparison, but cannot be used to identify firearms uniquely because this class of features will exist across many firearms which use similar firing pins. On the other hand, the micro-features, or the surface roughness, represent the individual characteristics, which arise from random variations in firearm manufacturing process and wear. These surface roughness features are unique between firearms, forming the basis for ballistics identification [13].

In order to preserve the critical individual characteristics and ensure accurate identification, a new method named differential profile extraction is developed. This simple method is to subtract the mean surface profile from original data profile. This subtraction obtains a higher fidelity result than simply using a filtering process on a section profile to remove low frequency form. The differential profile extraction is actually equivalent to separating the roughness component from the form and waviness of the area rather than that from the cross-section profile. Figure 7 (c) shows the subtraction result between profiles in Figs. 7 (a) and (b). 

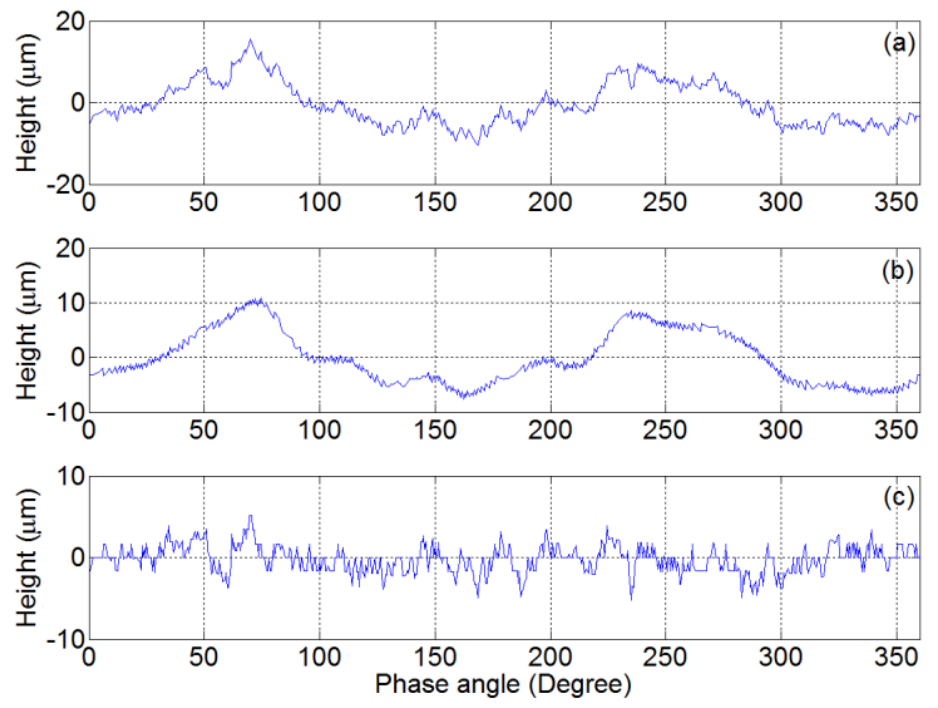

Fig. 7. Differential profile extraction: (a) profile extracted from the original topography; (b) profile extracted from the mean surface; (c) differential profile extraction.

Figure 8 shows a concatenated set of differential extracted profiles, after slicing using a vertical interval of $0.2 \mu \mathrm{m}$ and converting with a polar coordinate transformation. As a result, a "profile wall" can be seen. The complete implementation of profile extraction is described in block diagram form in Fig. 9.

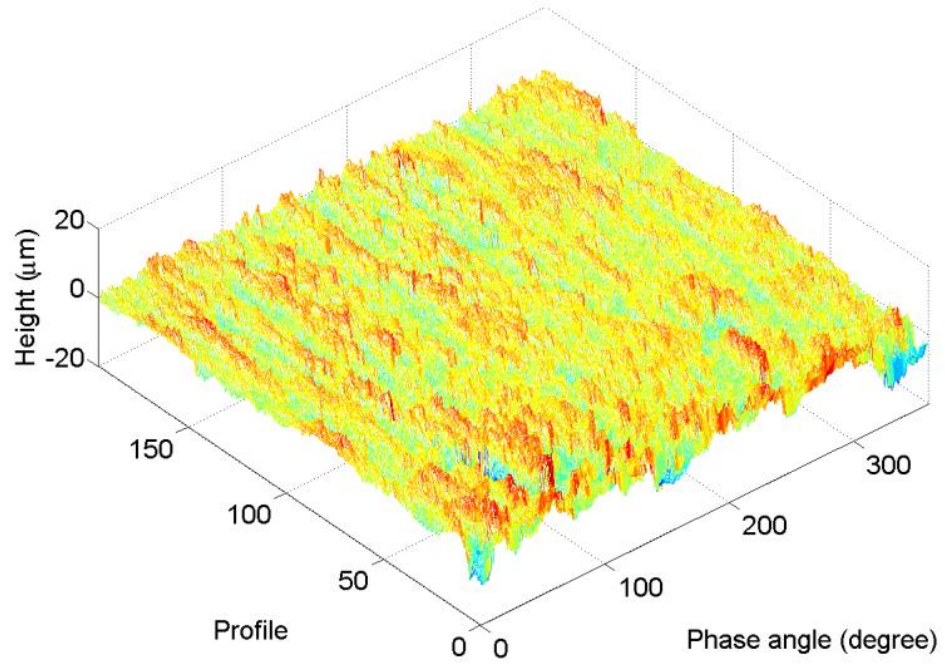

Fig. 8. Sampled point (Rotated to horizontal position). 


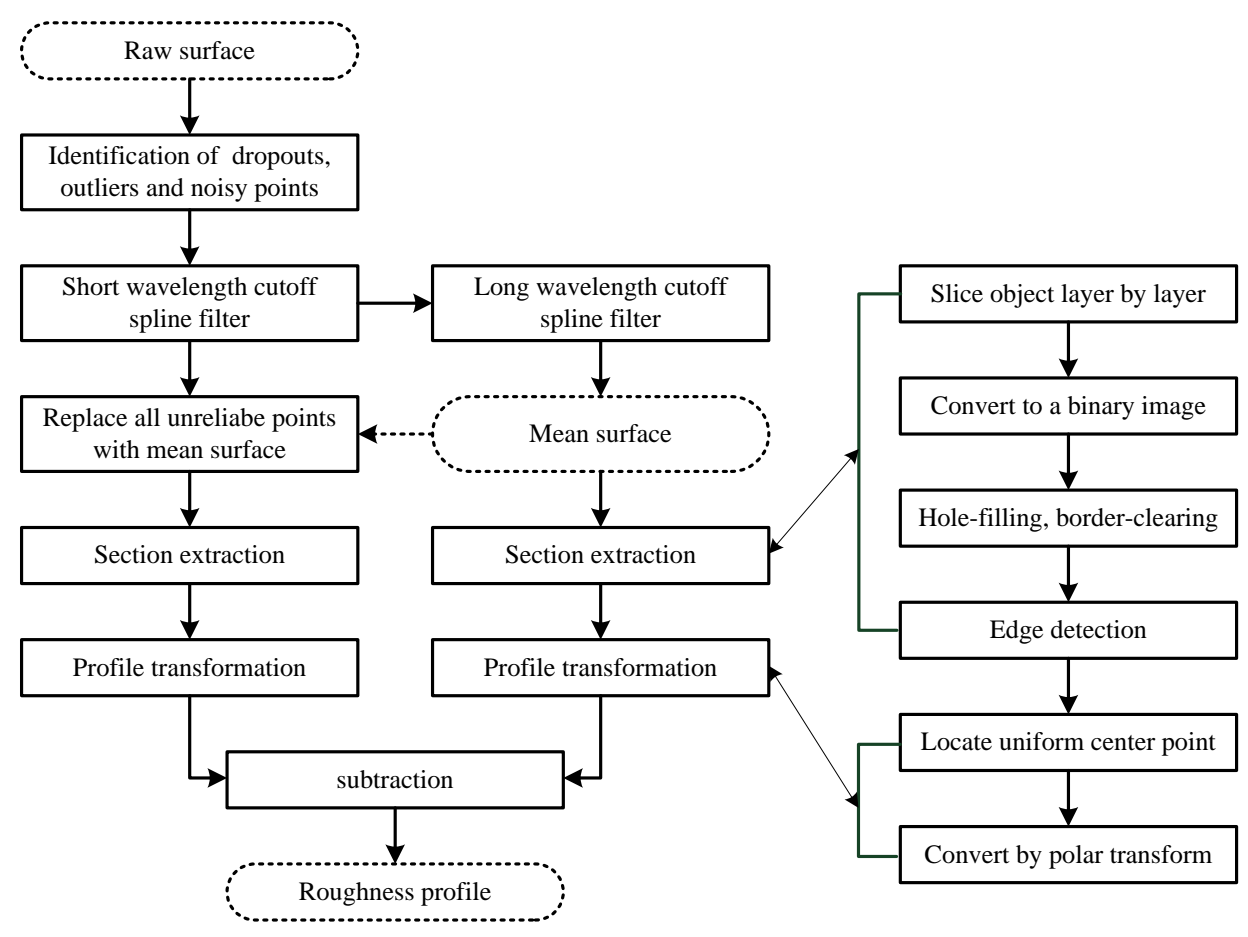

Fig. 9. Block diagram for producing a differential profile from a raw surface.

\subsection{Congruent matching algorithm}

Theoretically, the profiles located at the same height of the firing pin impressions fired from the same firearm should have high similarity in their roughness component. In practice, the quality of the impressions involves a number of random factors, which may cause minor changes to the micro-topography of the impressions. Hence, it is suggested to take an average of the adjacent parallel profiles to generate an averaged profile for more accurate profile correlations.

Since all the sampled firing pin impressions are measured regardless of their initial angular position, the resulting topographies of these impressions and the calculated profiles cannot be assumed to have any initial angular relationship. Hence, during the calculation of cross-correlation, one of the correlated profiles from the evidence image should be shifted cyclically to fit the other from the reference image and to find where $C C F_{\max }$ is located. In addition, the depth of the impression may vary from firing to firing, therefore the profiles of the reference image are correlated with profiles of the evidence image at various heights to take into account of the vertical variability. 
If the correlated cartridge pair is fired from the same firing pin, there should be the same phase angle $\theta$ where each profile of the reference image will be highly correlated with the corresponding (in height) profile of the evidence image. Collecting the distribution of registration angles that produce the maximum correlation value $C C F_{\max }$ for each of the profile pairs allows the optimum phase angle $\theta$ to be identified. As a result, three identification parameters are proposed to help this searching procedure and to determine the congruency of the pairwise correlated profiles: $C C F_{\max }$, phase angle $\theta$, and vertical section shift in height $\triangle P$. The tolerable range of $C C F_{\max }$ between correlated profiles may be variable depending on the pistol brands and cartridge materials. Hence it is unnecessary to design the tolerance excessively rigid on this parameter, a relatively loose threshold value is able to help collect a sufficient matching result. Similarly, the horizontal phase angle $\theta$ range and shifts in the vertical section shift $\Delta P$ where the best $\mathrm{CCF}$ is obtained between the correlated profiles array help to determine the "congruency" of the correlated profile distribution patterns.

When the values of these parameters and their statistical distributions are collected for analysis, we derive the number of potentially congruent profile pairs as the CMX number and use it as key parameter for a conclusive identification or exclusion.

\section{Experiments and results}

In order to validate the proposed CMX method, 40 cartridge cases of three brands fired from 10 pistols of three manufacturers are used for tests. The set of 40 cartridges are selected from the previous NIST Ballistics Imaging Database Evaluation (NBIDE) project [14]. Correlation program was developed using the commercially available MATLAB software environment [15]. Image to image comparisons within this test set includes 60 known-matching (KM) and the 720 known-non-matching (KNM) image pairs for a total of 780 correlations.

In this experiment, each impression is trimmed to be a $40 \mu \mathrm{m}$ height bowl relative to the 
bottom of its mean surface, so the width and length of the transformed profile wall are 196 slices (with a slice interval of $0.2 \mu \mathrm{m}$ ) and 720 angular data points respectively as shown in Fig. 8. The number of profiles averaged along the vertical dimension is selected to be four, to give a set of 49 profiles (with a vertical profile interval of $0.8 \mu \mathrm{m}$ ) per casing to be used in the identification algorithm.

Figure 10 (a) shows an example of a CMX angular distribution between two KM impressions. The three thresholds of the identification parameters are selected as: $C C F_{\max }=$ 0.25 , phase shift range is set to be $13.5^{\circ}$, and vertical shift range is 5 profile intervals, or $\Delta P$ $=5(4 \mu \mathrm{m})$. As expected, the phase angles where $C C F_{\max }$ is found for most profile sections are located in a limited angular region. Figure 10 (b) illustrates it by a new distribution calculated using a moving summation with a window equivalent to the threshold of $\theta$ $\left(13.5^{\circ}\right)$. This new distribution provides a count for how many profile sections are registered within $13.5^{\circ}$ of a given angle. The peak of this figure corresponds to the maximum CMX number and the angle where the peak occurs corresponds to the phase registration angle $\theta$.
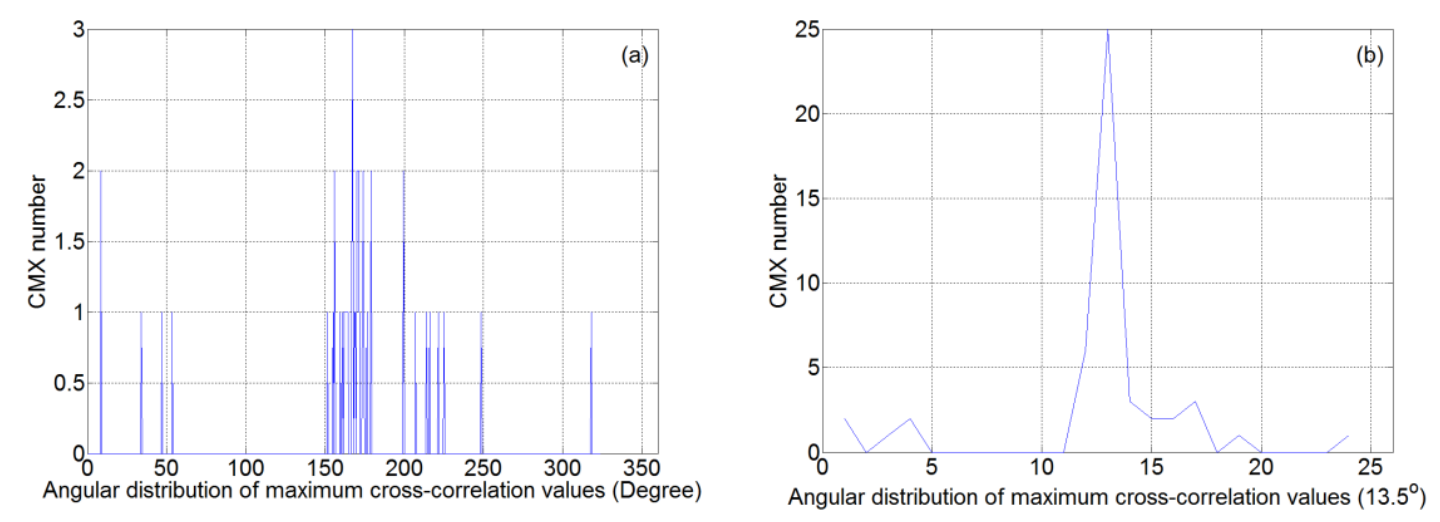

Fig. 10. CMX distribution at phase registration positions: (a) the CMX distribution of a correlated KM profile pair; (b) the CMX distribution calculated using a moving summation with a window equivalent to the threshold for $\theta\left(13.5^{\circ}\right)$.

For all the 40 cartridges, CMX distribution histograms for both KM and KNM groups are calculated using the parameters above and the results are summarized in Fig. 11. It can be seen that the KM and KNM group distributions are basically separated, but the separation is 
relatively small which needs to be improved.

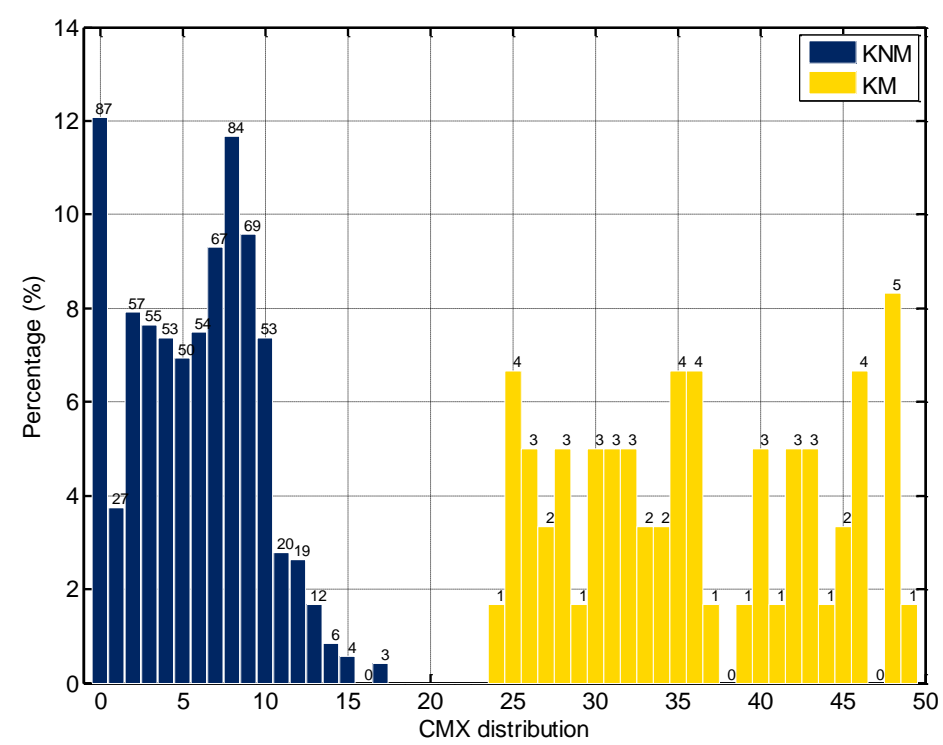

Fig. 11. Distribution of CMX scores for the test set of firing pin impressions.

It is possible to improve the separation of the distributions by computing the difference between main and second peaks of the CMX distribution as shown in Fig. 10 (b) [6]. The second peak of the distribution should be relatively close to the primary peak for KM pairs, whereas any peaks in the CMX distribution for KNM pairs will be randomly distributed [6]. By selecting the CMX number between the primary and second peaks of the distribution, this difference can be used to further separate the two distributions. The new CMX distribution histograms are shown in Fig. 12, in which the KM and KNM distributions are well separated with a larger gap. Hence, we may define a determinant difference between main and second peaks of CMX as another identification parameter CMX'. Obviously, larger values of $\mathrm{CMX}^{\prime}$ correspond to a higher degree of matching between firing pin impressions. Utilizing the center of the distribution gap at $\mathrm{CMX}^{\prime}=17$, a $\mathrm{CMX}^{\prime}$ selection criterion can be chosen to differentiate between identifications and exclusions. Assuming criterion $\mathrm{CMX}^{\prime}=17$, all the $60 \mathrm{KM}$ and $720 \mathrm{KNM}$ image pairs are identified correctly. 


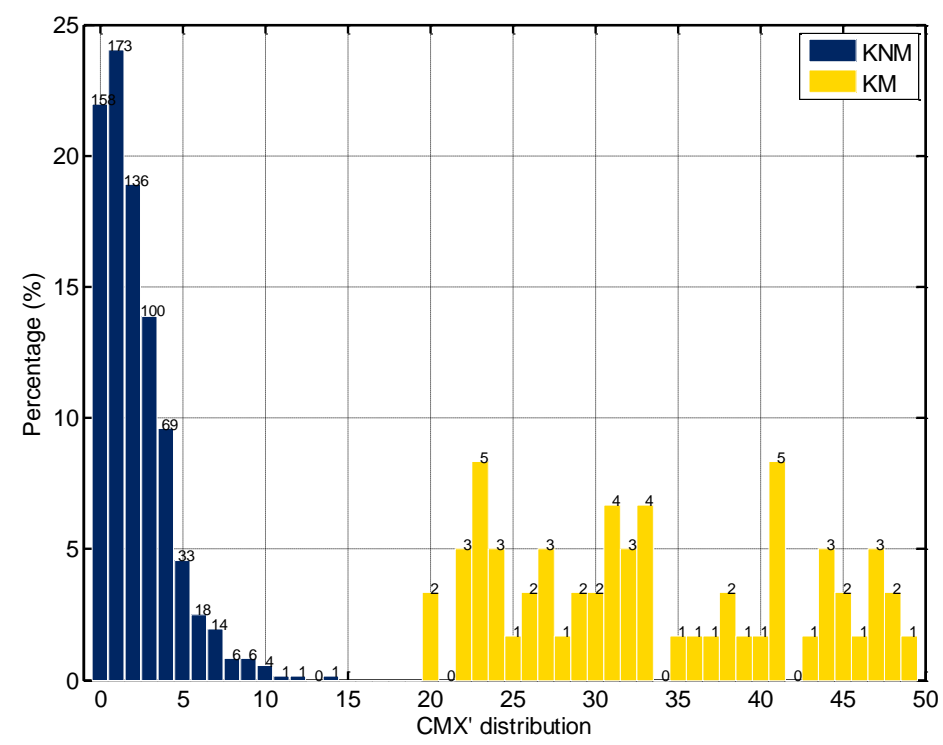

Fig. 12. Distribution of $\mathrm{CMX}^{\prime}$ scores for the test set of firing pin impressions.

Figure 13 shows the distribution of the average CMX pairs (in \%) of the $720 \mathrm{KNM}$ and $60 \mathrm{KM}$ image pairs with the variation of the profile heights presented by the number of vertical sections. The values that are labeling the bars display the average number of valid CMX pairs at each section of KM/KNM image group respectively. The result indicates that the relative CMX pairs of KM images are significantly larger than that of KNM images. From the summation over all layers, the average CMX number of for KM images approaches 38, but the average CMX number of the KNM group is only 5.8.

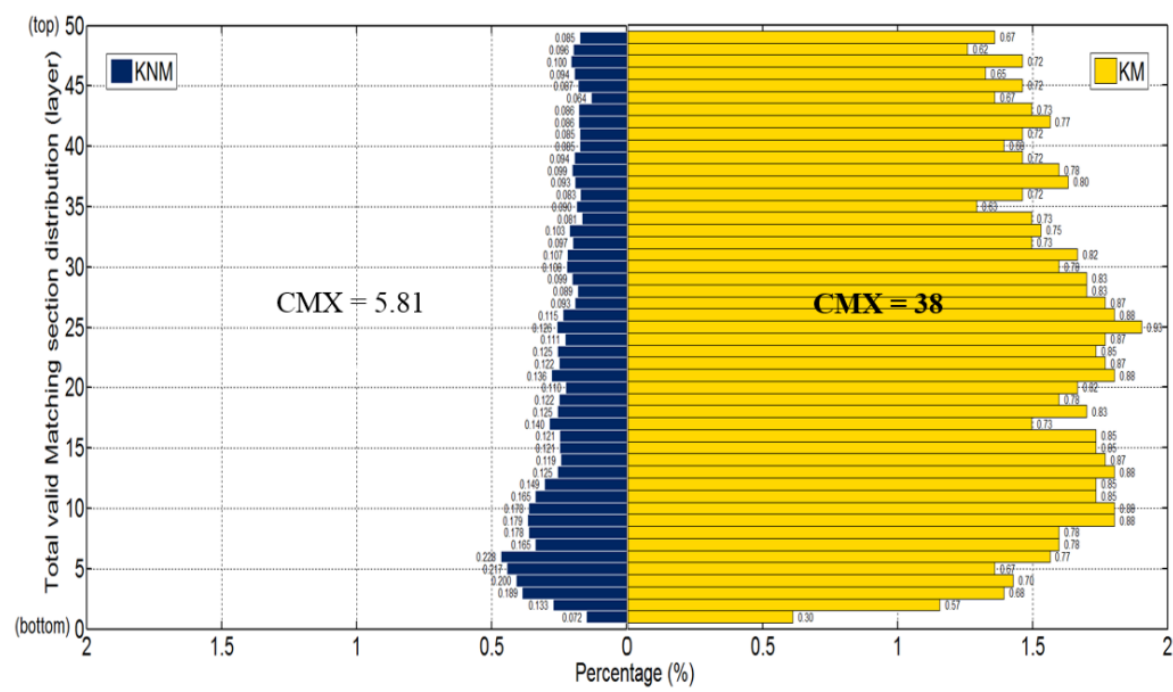

Fig. 13. Average CMX numbers for $60 \mathrm{KM}$ image pairs and $720 \mathrm{KNM}$ image pairs, and their distribution by cross-sections. 


\section{Discussion and future work}

Although the initial validation tests for the proposed CMX method doesn't show overlap between the KM and KNM distributions (Fig. 12), there are some questions to be discussed for future improvement of the CMX method.

\subsection{Image depth}

The image depth must be carefully selected—not too high, neither too low. If the image depth is too high, it may include too much dropout and outlier information into correlation (see Figs. 2 and 3), that may decrease the correlation accuracy. On the other hand, from Figure 13, it can be seen that the CMX scores of the KM image pairs show roughly even distribution among the vertical sections, but dramatically decrease at the bottom. That may be from the profile distortion at the bottom sections: the differential profiles are extracted from horizontal sections; however, the profile features (peaks, valleys...) at the bottom sections are mainly located in vertical direction. As a result, the profile extraction in the horizontal direction may cause large profile deformation. From Figure 8, it can be seen that the significant increases of peak heights and valley depths, or profile deformations, are mainly happened at the bottom sections.

It is suggested: (1) to eliminate the bottom sections from CMX correlations; (2) to use the congruent matching cells $(\mathrm{CMC})$ method $[3,5,6]$ for correlation of the firing pin images at the bottom sections, and combine the CMC result with the CMX result for ballistics identification of the firing pin images.

\subsection{Identification criterion}

It is necessary to conduct multiple tests using firing pin images from multiple gun and ammo manufacturers to develop an identification criterion for CMX method to be used for ballistics identification of firing pin images.

\subsection{Error rate estimation}

The CMX method is a branch of the congruent matching family proposed at NIST for 
ballistics identifications [2,3]. The CMX method is based on pairwise section correlations with multiple identification parameters. That makes it possible to develop an error rate procedure for reporting the false positive and false negative error rates based on the total section numbers $N$, the qualified CMX number, and the statistical distribution of the three identification parameters: cross-correlation function $C C F_{\max }$, registration angle $\theta$ and the section variation $\Delta P$ for both the $\mathrm{KM}$ and $\mathrm{KNM}$ image pairs. This procedure has been successfully used at NIST for reporting error rates of breech face correlations of cartridge case images using the CMC method [4]. It could also be used for reporting error rates of firing pin correlations using the CMX method.

\section{Conclusion}

The firing pin impression on a cartridge case is one of the most important signatures for firearms identification. In this paper, a novel identification algorithm is proposed for firing pin impressions based on topography measurements using confocal microscopy and the proposed congruent matching cross-sections (CMX) method.

For the special convex shape of firing pin impression, a cross-section slice process is developed by slicing the impression images layer by layer. All sections are converted to a set of profiles based on a polar coordinate transformation. Three identification parameters with thresholds are developed for quantifying profile similarity by cross-correlation function $C C F_{\max }$, and for quantifying congruency of profile distribution patterns by phase angle $\theta$ and section location $\Delta P$. If the correlated cartridge pair is fired from the same firing pin, there would be a closer phase registration angle at a closer section height where each profile of the evidence image is highly correlated with the corresponding profile of the reference image. That profile pair is considered as a CMX. If the CMX number is larger than a statistically determined identification criterion, the correlated cartridge pair is considered from the same firearm.

The CMX method and the proposed identification algorithm are validated by 780 
correlations of a set of 40 cartridge cases of three brands fired from 10 guns of three manufacturers. These topographies are correctly identified based on the three parameters. Even though the data set used in this study was limited and was collected from a limited type of firearms and ammos, it is practical to extend the method to a larger data set. In addition, we also identified that texture information on the bottom is less useful for the proposed CMX method than that on other parts of the firing pin impression. Hence, an improvement of combining CMX and some other algorithm (such as $\mathrm{CMC}$ ) for bottom sections may enhance the accuracy of firing pin identifications.

\section{References}

[1] Strengthening forensic science in the United States--a path forward. The National Research Council, (2009) 153-155, 184.

[2] Author. Proposed "NIST ballistics identification system" based on 3D topography measurements on correlation cells. AFTE Journal, 45(2) (2013) 184-194.

[3] Author. Proposed "congruent matching cells (CMC)" method for ballistics identification and error rate estimation. AFTE Journal, 47(3) (2015).

[4] Author. Estimating error rates for firearm evidence identifications in forensic science. Passed NIST review, to be submitted to Forensic Science International for review and publication.

[5] Author. Validation tests for the congruent matching cells (CMC) method using cartridge cases fired with consecutively manufactured pistol slides. AFTE Journal, 45(4) (2013) 361-366.

[6] Author. An improved algorithm of congruent matching cells (CMC) method for firearm evidence identifications. Journal of Research of NIST, 120 (2015) 102-112.

[7] David Casasent, Demetri Psaltis. Position, rotation and scale invariant optical correlation. Applied optics, 15(7) (1976) 1795-1799.

[8] Author. Automatic identification of bullet signatures based on consecutive matching striae (CMS) criteria. Forensic Sci. Int., 231 (2013) 137-141.

[9] Author. An areal isotropic spline filter for surface metrology. Journal of Research of NIST, 120 (2015) 64-73.

[10] ISO/TS 16610-31. Geometrical product specifications (GPS)-filtration-part 31: Robust profile filters: 
Gaussian regression filters; 2006.

[11] Soille Pierre. Morphological Image Analysis: Principles and applications. Springer-Verlag, (1999) 173174.

[12] Rein van den Boomgaard, Richard van Balen. Methods for fast morphological image transforms using bitmapped images. CVGIP, 54(3) (1992) 252-258.

[13] Scientific working group for firearm and toolmarks (SWGGUN). The foundations of firearm and toolmark identification, http://www.swggun.org (accessed July 6th, 2015).

[14] Author. Surface topography analysis for a feasibility assessment of a National Ballistics Imaging Database. NISTIR 7362, NIST, 2007.

[15] MATLAB Release 2013a. The MathWorks, Inc., Natick, Massachusetts, United States. 